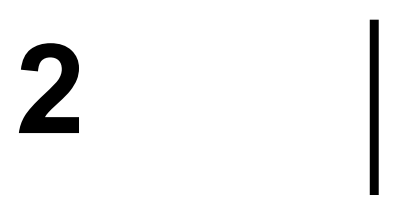

\section{La Mémoire Humaine et ses substrats cérébraux}

\section{Francis Eustache et Armelle Viard}

Inserm-EPHE-Université de Caen U1077 Neuropsychologie et Imagerie de la Mémoire Humaine

\title{
Abstract
}

Francis Eustache and Armelle Viard invite us to take an interest in the research on human memory. Francis Eustache recalls how pathological memory disorders have gradually, and very strongly since the $1960 \mathrm{~s}$, been a source of information in the development of theoretical models of human memory. He develops an analysis of the genesis and evolution of these models and the concepts which underpin them; he ends with a brief introduction to the MNESIS model that he developed with his team, showing its relationships with the previous models. Armelle Viard deals specifically with the running of "autobiographical" memory and the structures involved in it, in comparison with "semantic" memory. She draws up a table of various experimental works which have been carried out on these themes, in particular using neuroimaging; she presents and discusses the results. She thus deals with how the reconstruction / recovery of a memory is carried out, the parameters which intervene in such processes and the brain regions involved. She ends with an analysis of autobiographical memory disorders in neurodegenerative pathologies such as Alzheimer's and semantic dementia. 


\section{Partie A : Neuropsychologie de la mémoire humaine Francis Eustache}

\section{Comment définit-on la mémoire ?}

\subsection{Introduction}

A partir des années 1960, les syndromes amnésiques ont constitué la source d'inférence principale pour élaborer des modèles théoriques de la mémoire humaine. Ils se caractérisent par une atteinte de la mémoire, d'origine organique, disproportionnée par rapport à d'autres troubles cognitifs. La recherche de dissociations entre capacités mnésiques perturbées et capacités mnésiques préservées devient le grand paradigme de la neuropsychologie de la mémoire (Eustache et Desgranges, 2012 ; Eustache et al, 2018 ; Eustache et Guillery-Girard, 2016).

L'exemple emblématique est la dissociation, mise en évidence chez le patient amnésique H.M. par Brenda Milner, psychologue canadienne, entre l'atteinte de la mémoire à long terme et la préservation de la mémoire à court terme. La dissociation inverse est rapportée par les psychologues britanniques Shallice et Warrington à propos du patient K.F., présentant une préservation de la mémoire à long terme et une réduction de l'empan auditivo-verbal. Cette opposition est confortée par de nombreuses données expérimentales, comme les effets de primauté et de récence qui se traduisent par le rappel préférentiel des mots du début et de la fin d'une liste reflétant respectivement la fonctionnalité de la mémoire à long terme et de la mémoire à court terme.

La notion de mémoire à court terme s'est complexifiée pour aboutir au concept de mémoire de travail à composantes multiples. La mémoire à long terme a également donné lieu à plusieurs fractionnements. Les études de patients amnésiques montrent en effet qu'en dépit de difficultés à acquérir volontairement des informations nouvelles, ils sont capables d'apprentissage ou témoignent par leur comportement de la rétention d'expériences antérieures, souvent à leur insu.

A partir des années 1970, la neuropsychologie de la mémoire connaît ainsi deux évolutions parallèles : l'élaboration du concept de mémoire de travail et le démembrement de la mémoire à long terme.

\subsection{Mémoire à court terme et mémoire de travail}

La mémoire de travail, définie par les psychologues britanniques Baddeley et Hitch en 1974, est un système responsable du traitement et du maintien temporaire des 
informations nécessaires à la réalisation d'activités aussi diverses que la compréhension, l'apprentissage et le raisonnement.

Elle est composée de deux sous-systèmes satellites de stockage (la boucle phonologique et le calepin visuo-spatial), coordonnés et supervisés par une composante attentionnelle, l'administrateur central. La boucle phonologique est responsable du stockage d'informations verbales, de leur manipulation et de leur rafraîchissement. Elle est constituée d'un registre phonologique de stockage passif, de capacité limitée et d'un processus d'autorépétition subvocale, la récapitulation articulatoire, permettant le rafraîchissement de l'information et la conversion d'un stimulus présenté visuellement en un code phonologique. Le calepin visuo-spatial est impliqué dans le stockage des informations spatiales et visuelles ainsi que dans la formation et la manipulation des images mentales.

L'administrateur central supervise et coordonne l'information en provenance des systèmes satellites et gère le passage de l'information vers la mémoire à long terme. Il joue un rôle dans la focalisation et le partage de l'attention, dans la sélection des informations en mémoire à long terme, dans la manipulation de ces informations et dans l'intégration en mémoire à long terme des nouvelles informations. Ainsi, la mémoire de travail n'est pas uniquement une voie de passage des entrées sensorielles en mémoire à long terme mais un espace de travail entre les données issues de l'environnement et les connaissances en mémoire à long terme.

Au début des années 2000, Baddeley a postulé l'existence d'un nouveau système temporaire de stockage, le buffer épisodique, chargé du stockage temporaire d'informations intégrées provenant de différentes sources. Il est contrôlé par l'administrateur central, qui récupère ces informations depuis les systèmes de stockage sous la forme de processus conscients, traite ces informations et, si nécessaire, les manipule et les modifie. Ce buffer est épisodique car il stocke des épisodes dans lesquels l'information est intégrée dans l'espace et le temps. Il se rapproche du concept de mémoire épisodique mais en diffère car il s'agit d'un système de stockage temporaire qui peut être préservé chez des amnésiques ayant des troubles de la mémoire épisodique.

\subsection{La mémoire à long terme}

L'une des distinctions les plus importantes concerne la mémoire épisodique et la mémoire sémantique, proposée initialement par le psychologue canadien Tulving en 1972. La mémoire épisodique est définie comme la mémoire des événements personnellement vécus, situés dans leur contexte temporo-spatial d'acquisition. Sa caractéristique fondamentale est de permettre le souvenir conscient d'une expérience antérieure : l'événement lui-même (quoi), le lieu (où) et le moment (quand) où il s'est produit. En plus de l'exactitude du souvenir de l'événement rappelé, ce qui caractérise cette mémoire est l'expérience subjective, l'impression de revivre l'événement. La récupération d'un souvenir en mémoire épisodique implique ainsi un «voyage mental dans le temps » associé 
à la conscience autonoétique (ou conscience de soi). Cette définition met l'accent sur la conjonction de trois idées : l'identité (ou self), la conscience autonoétique et le temps subjectif. La situation du patient amnésique K.C., victime de plusieurs traumatismes crâniens, décrit par Tulving, permet de comprendre à quel point l'absence de mémoire épisodique et de conscience autonoétique engendre une impression de vide sans retour vers le passé ni projection dans le futur.

La mémoire sémantique a d'abord été définie comme la mémoire des mots, des concepts, des «connaissances du monde », indépendamment de leur contexte d'acquisition. Elle s'est vue ensuite attribuer la notion de conscience noétique ou conscience de l'existence du monde, des objets, des événements et de diverses régularités. La mémoire sémantique permet ainsi une conduite introspective sur le monde. Le concept comprend également les connaissances générales sur soi (ou sémantique personnelle).

L'opposition entre mémoire déclarative et mémoire procédurale, proposée dans les années 1980 par le neuroscientifique américain Squire et ses collaborateurs, se situe à un autre niveau et correspond à la distinction, plus ancienne, entre mémoire et habitude. L'information stockée en mémoire déclarative est facilement verbalisable et accessible à la conscience. Les représentations peuvent être générales (sémantiques) ou spécifiques (épisodiques). La mémoire procédurale permet d'acquérir des habiletés progressivement, au fil de nombreux essais, de les stocker et de les restituer sans faire référence aux expériences antérieures. Elle s'exprime dans l'activité du sujet et ses contenus sont difficiles à verbaliser. La mémoire procédurale est une mémoire automatique et difficilement accessible à la conscience.

Une autre distinction, opposant mémoire explicite et mémoire implicite, a été proposée dans sa version moderne par le psychologue américain Schacter, dans les années 1980. Certains résultats ont conduit à la proposition, par Tulving et Schacter en 1990, d'un nouveau système de mémoire, le système de représentations perceptives. La mémoire implicite est mise en jeu quand des expériences préalables modifient la performance dans une tâche qui ne requiert pas le rappel conscient de ces expériences. Par exemple, le fait de voir une image une première fois facilite l'identification ultérieure de cette image, y compris si elle est présentée sous une forme dégradée, et ceci sans que le sujet ait conscience de faire appel à sa mémoire. Au contraire, la mémoire explicite fait référence aux situations dans lesquelles un sujet rappelle volontairement des informations stockées en mémoire.

\subsection{Des concepts aux modèles}

Malgré la pléthore de qualificatifs associés au mot " mémoire », seules certaines composantes de la mémoire ont accédé au statut de système de mémoire, chacun ayant sa relative indépendance, ses caractéristiques propres et ses règles de fonctionnement. En accord avec la proposition de Tulving, nous retenons une organisation de la mémoire 
formée de cinq systèmes : la mémoire de travail, la mémoire procédurale, la mémoire perceptive, la mémoire sémantique et la mémoire épisodique. Nous reprenons les terminologies proposées par Tulving et par d'autres auteurs, notamment Squire et Baddeley qui ont laissé leur empreinte sur ces différents concepts.

MNESIS (pour Modèle NEoStuctural InterSystémique ; Eustache et al, 2016 ; voir figure 1) intègre les éléments les plus robustes des conceptions multi-systèmes proposées par ces auteurs, tout en spécifiant davantage les relations entre les systèmes et les apports récents de la neuroimagerie. Les trois systèmes de représentation à long terme (mémoire perceptive, mémoire sémantique, mémoire épisodique) sont présentés en respectant l'organisation hiérarchique proposée par Tulving. Les traces mnésiques transitent par les mémoires perceptives avant d'accéder, éventuellement, au statut de représentations sémantiques (dans la mémoire sémantique) et, éventuellement, à celui de souvenirs (dans la mémoire épisodique). Cette organisation rend compte du fait que des patients amnésiques (avec un trouble de la mémoire épisodique) continuent de former des connaissances sémantiques.

A gauche de ces trois systèmes de représentation figurent deux flèches qui doivent être considérées comme des rétroactions. L'une (allant de la mémoire épisodique à la mémoire sémantique) désigne le processus de sémantisation des souvenirs et insiste sur le fait que les souvenirs font l'objet d'un processus de sémantisation au fil du temps. La seconde flèche (de la mémoire épisodique à la mémoire perceptive) met l'accent sur les phénomènes de reviviscence, conscients et inconscients, indispensables à la consolidation mnésique. Il s'agit de processus très divers allant de la ré-évocation de la scène initiale émaillée de détails sensoriels à des mécanismes moins contrôlés se produisant pendant des rêveries ou certains stades de sommeil. Ces rétroactions soulignent le caractère dynamique et reconstructif de la mémoire et leur corollaire, les transformations de la trace mnésique et la formation possible de faux souvenirs.

Au centre de la figure se trouve la mémoire de travail avec, d'une part, les composantes du modèle «classique » de Baddeley : administrateur central, boucle phonologique, calepin visuo-spatial et, d'autre part, le buffer épisodique. Le buffer épisodique place la mémoire au centre du psychisme et doit être rapproché de la notion de conscience de soi, qui donne au sujet une impression subjective de soi dans le temps et qui préside au sentiment d'intégrité et de continuité.

A droite du modèle est représentée la mémoire procédurale intégrant les supports d'habiletés motrices, perceptivo-motrices et cognitives. Les interactions entre ce système d'action et les systèmes de représentation sont matérialisées par les différentes flèches. Les liens avec la mémoire perceptive sont privilégiés pour la mémoire procédurale perceptivomotrice, et avec les systèmes déclaratifs pour la mémoire procédurale cognitive. Dans tous les cas, les interactions avec les systèmes de représentation (y compris la mémoire de travail) sont particulièrement importantes lors de la phase d'apprentissage procédural. 


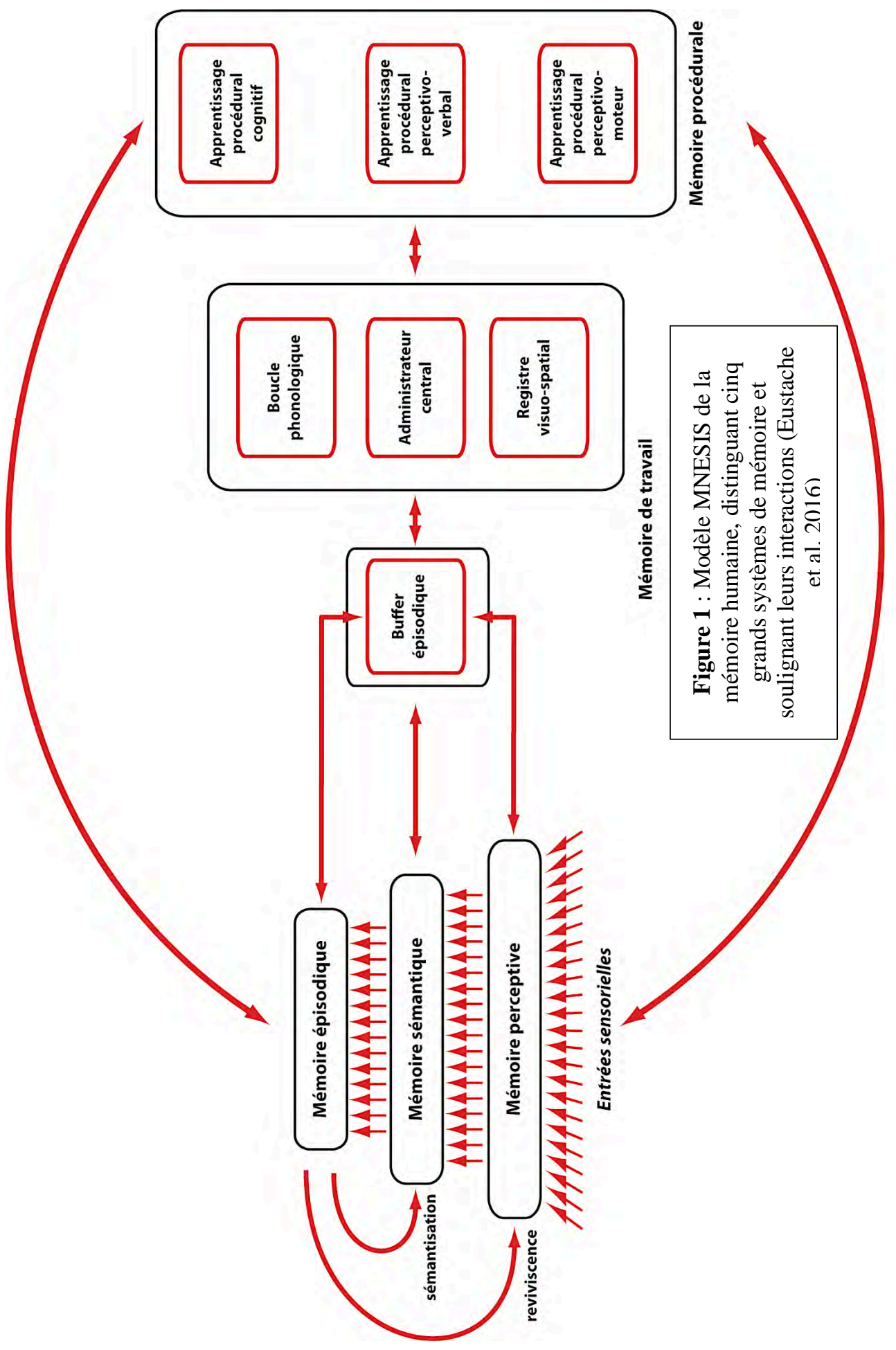




\section{Partie B : Neuroimagerie de la mémoire humaine Armelle Viard}

\section{Mémoire autobiographique}

\subsection{Définition}

La mémoire autobiographique est un domaine d'investigation qui a donné lieu à des travaux très variés. La mémoire autobiographique permet de voyager dans le temps subjectif et donne l'impression de revivre mentalement les détails phénoménologiques des événements du passé. Toutefois, les représentations de la mémoire autobiographique ne sont pas uniquement épisodiques : nombre d'entre elles sont sémantiques. Au total, cette mémoire correspond à la mémoire à long terme qui permet d'encoder, de stocker et de récupérer des informations dont le Self est le sujet central : il s'agit, en raccourci, de la «mémoire du Self ».

\subsection{Modèle de Conway}

Un modèle d'organisation de la mémoire autobiographique a été proposé par Conway et collaborateurs (Conway et Pleydell-Pearce, 2000). Conway considère que les souvenirs autobiographiques mettent en jeu des processus mnésiques complexes et reconstructifs : le souvenir est construit de façon dynamique à partir de quatre types de représentations organisées hiérarchiquement, du plus général au plus spécifique : le schéma historique personnel, les périodes de vie, les événements généraux et les détails spécifiques. Le schéma historique personnel, les périodes de vie et les événements généraux constituent les connaissances autobiographiques de base («autobiographical knowledge base ») et les détails perceptivo-sensoriels d'événements spécifiques sont regroupés dans ce que les auteurs appellent le système de mémoire épisodique. 
Plus spécifiquement, le schéma historique personnel (« life-story schema ») représente le niveau le plus global et correspond à des généralisations de thèmes dominants ou de chapitres de vie (par exemple, « ma vie en tant que femme anglaise du 21ème siècle » ou «ma carrière en tant que professeur dans une université américaine »). Les périodes de vie ( «life-time periods») constituent un niveau abstrait et étendu dans le temps, mesuré en années ou en décennies (e.g., la période de «l'école maternelle » se caractérise par un contenu thématique - les images génériques des professeurs, des salles de classe - et par une durée avec un début et une fin spécifiés). Les événements généraux ("general events ») sont plus spécifiques et en même temps plus hétérogènes que les périodes de vie et se mesurent en jours, en semaines ou en mois (e.g. « les week-ends à la campagne », «mon week-end en Italie »). Les détails perceptivo-sensoriels d'événements spécifiques (« event specific knowledge »), mesurés en secondes, en minutes ou en heures, correspondent au registre phénoménologique de la trace mnésique (images, sentiments, odeurs...). Ces détails sont rapidement oubliés, à moins d'être répétés ou associés à des connaissances générales.

À chaque étape du processus mnésique (encodage, stockage, récupération), ces quatre formes de représentation sont organisées dans la mémoire à long terme sous l'influence du modèle d'intégrité personnelle du sujet (ou «self»). À l'encodage, les événements sont retenus s'ils sont en accord avec les buts actuels du self (Conway et Pleydell-Pearce, 2000). Lors de la récupération, la reconstruction du souvenir est soumise à un processus central de contrôle, modulé par le self. Le processus de récupération est cyclique et dépend de l'administrateur central de la mémoire de travail.

\subsection{Réseau cérébral}

\subsubsection{Régions sous-tendant la reconstruction de souvenir}

La récupération de souvenirs autobiographiques peut se faire selon deux modes : 1/ la récupération directe qui se produit de façon automatique et involontaire et $2 /$ la récupération générative qui est intentionnelle et contrôlée. Le processus de récupération directe ( «direct retrieval») consiste en l'accès involontaire aux détails spécifiques se faisant souvent en réponse à des indices très particuliers (une odeur, une saveur, une mélodie). Ce type de récupération n'est pas contrôlé par des processus exécutifs superviseurs. Le processus de récupération générative («generative retrieval») consiste en un processus complexe qui exige que le sujet se trouve dans un mode de récupération ( « retrieval mode » caractérise l'état dans lequel le sujet se trouve quand il cherche à se rappeler volontairement un événement personnel). Ce processus de reconstruction se décompose en trois phases : l'élaboration d'indices qui permettra d'initier un contexte de recherche, la recherche d'un événement dans ce contexte, la vérification du résultat de l'étape précédente. Si le résultat n'est pas satisfaisant, le processus recommencera en utilisant l'information issue de la deuxième étape comme un nouvel indice. 
Ce modèle met l'accent sur le rôle prédominant du lobe frontal dans la récupération autobiographique. Le contrôle est modulé par les buts actuels du sujet. Un souvenir récupéré est donc un profil d'activation particulier et stable des quatre types de connaissances, maintenu de façon transitoire en mémoire de travail. Ce processus rend compte de la déformation des souvenirs puisque le souvenir est encodé puis reconstruit et interprété à chaque fois en fonction du self actuel du sujet. Ainsi, l'événement encodé et récupéré est une interprétation propre au sujet.

Dans une étude réalisée en électroencéphalographie (EEG), Conway et collaborateurs ont proposé à des participants une tâche qui consistait à rechercher des souvenirs autobiographiques à partir de mots indices (phase de récupération de 5 secondes), puis à maintenir chaque souvenir en mémoire (phase de maintien de 5 secondes) et enfin à se préparer pour la présentation du mot indice suivant (phase de préparation de 5 secondes). Les résultats de cette étude EEG sont résumés dans la Table 1. Conway a proposé une interprétation de ces résultats: dans la phase de récupération, le déplacement des activations des régions antérieures aux régions postérieures reflèterait l'initiation (lobe frontal gauche), puis le résultat du processus de contrôle lorsque le souvenir est reconstruit (régions postérieures). Les régions occipitales semblent particulièrement impliquées pour les souvenirs vivaces, importants ou spécifiques. Par ailleurs, l'activation plus faible du lobe frontal droit traduirait l'accès à la connaissance de base (sémantique personnelle). Dans la phase de maintien, les activations latéralisées à droite indiqueraient les régions de stockage des connaissances autobiographiques. La phase de préparation est considérée comme une phase d'inhibition du processus de récupération. Ces résultats confortent le modèle de la mémoire autobiographique de Conway.

Table 1 : Principaux résultats du décours temporel des activations cérébrales au cours d'une tâche d'évocation de souvenirs autobiographiques.

\begin{tabular}{|l|l|}
\hline Phase de récupération & $\begin{array}{l}\text { Hémisphère gauche (lobe frontal et temporal antérieur) et } \\
\text { régions postérieures bilatérales (temporales et occipitales) }\end{array}$ \\
\hline Phase de maintien & $\begin{array}{l}\text { Hémisphère droit (lobe frontal et régions postérieures } \\
\text { temporales et occipitales) }\end{array}$ \\
\hline Phase de préparation & $\begin{array}{l}\text { Hémisphère droit (régions pariétales) } \\
\text { Désactivation des régions temporales et occipitales }\end{array}$ \\
\hline
\end{tabular}

Une méta-analyse de données issues d'études en imagerie par résonance magnétique fonctionnelle (IRMf) a permis d'établir la localisation précise des activations cérébrales observées pendant le rappel autobiographique. A l'inverse de l'EEG, l'IRMf a une haute résolution spatiale qui permet de localiser précisément les régions cérébrales activées lors 
d'une tâche cognitive particulière. Svoboda et al. (2006) ont analysé les résultats de 24 études en IRMf focalisées sur le rappel en mémoire autobiographique et montrent que la récupération d'un souvenir active un réseau cérébral ( core network» ou réseau cérébral principal), latéralisé à gauche et comprenant les cortex préfrontal médian et ventrolatéral, les cortex temporal médian (incluant l'hippocampe et le gyrus parahippocampique) et temporal latéral, les cortex retrosplénial et cingulaire postérieur, la jonction temporopariétale et le cervelet. Des régions moins fréquemment retrouvées sont également mentionnées.

Parmi ces études en IRMf, certaines ont permis de préciser la localisation cérébrale sous-tendant une étape particulière du rappel autobiographique, et ce de manière plus précise que les études en EEG. Steinvorth et al. (2006) ont effectué une étude en IRMf en demandant aux participants de récupérer des souvenirs autobiographiques. Les auteurs ont comparé la phase initiale de recherche des souvenirs à la phase de réminiscence et ont observé l'activation du cortex préfrontal ventro-latéral (aires de cérébrales Brodmann 44, $45,47)$. Cette région serait donc spécifiquement activée lors de la phase initiale de recherche d'un souvenir. Cabeza et al. (2004) se sont plutôt intéressés aux régions activées lorsqu'on évoque un souvenir propre à soi par rapport à un événement non personnel. Ils ont demandé à des étudiants de prendre des photos d'endroits précis du campus universitaire et ont comparé ces photos à celles prises par un autre participant. Les auteurs ont montré que les photos prises par soi-même activaient plus le cortex préfrontal médian (aires de Brodmann 10,32) par rapport à des photos prises par un autre individu. Cette région serait donc spécialement dédiée au traitement d'informations relatives à soi.

\subsubsection{Régions influencées par la qualité des souvenirs : émotion}

D'autres études en IRMf ont évalué le rôle de la qualité des souvenirs rappelés et les régions cérébrales associées. L'émotion est une caractéristique phénoménologique importante des souvenirs autobiographiques persistants et vivaces. En effet, une étroite relation fonctionnelle existe entre mémoire et émotion comme le soulignent des travaux montrant un rappel préférentiel de souvenirs autobiographiques émotionnels. Les souvenirs émotionnels perdurent et gardent leur vivacité, ce qui semble manquer aux autres souvenirs, et sont mieux mémorisés que des événements neutres.

$\mathrm{Au}$ plan anatomique, le complexe amygdalien joue un rôle important dans le traitement d'informations émotionnelles, comme le confirment des études en neuroimagerie. Cette structure est connectée avec des régions fronto-temorales impliquées dans la mémoire autobiographique. Certaines études d'IRMf ont comparé le rappel de souvenirs émotionnels à celui de souvenirs sémantiques (Greenberg et al., 2005) ou moins émotionnels (Viard et al., 2010) et ont confirmé l'implication de l'amygdale. Grâce à des analyses de connectivité fonctionnelle, ces études ont également montré l'existence d'une co-activation entre l'amygdale et l'hippocampe pendant le rappel de souvenirs émotionnels. 


\subsubsection{Régions influencées par la qualité des souvenirs : imagerie visuelle mentale}

L'imagerie mentale visuelle joue également un rôle important dans la récupération en mémoire autobiographique et serait liée à l'épisodicité du souvenir : l'imagerie visuelle augmente le rappel de détails spécifiques et l'expérience subjective du souvenir. L'imagerie visuelle serait le meilleur facteur de spécificité par rapport à d'autres indices (olfactifs, tactiles, auditifs, moteurs). L'imagerie est un indice de récupération efficace et économique contenant des informations génériques sur les principaux personnages, le lieu et l'organisation temporelle de l'événement vécu, ces indices pouvant être utilisés lors de la récupération. La quantité de détails phénoménologiques présents lors du rappel autobiographique pourrait refléter son exactitude (Conway and Pleydell-Pearce, 2000) et distinguer les événements vécus des événements imaginés.

Gardini et al. (2006) se sont intéressés au rôle de l'imagerie visuelle mentale dans le rappel autobiographique. Suite à la présentation d'un mot cible (e.g., voiture), les participants devaient générer une image visuelle mentale en rapport avec le mot présenté (e.g., ma voiture) ou dans un contexte dépersonnalisé (e.g., une voiture). Les auteurs ont montré que la génération d'images mentales relatives à soi activait préférentiellement le cunéus, le précuneus et le gyrus parahippocampique par rapport à la génération d'images impersonnelles. Ainsi, ces régions auraient un rôle spécifique dans l'imagerie visuelle mentale d'items relatifs à soi.

\subsubsection{Régions influencées par l'ancienneté du souvenir (récent vs. ancien)}

Différents travaux ont montré que l'effet de l'âge du souvenir est hétérogène selon les systèmes de mémoire (Eustache et al., 1998). La mémoire épisodique est particulièrement sensible à l'effet de l'âge, contrairement à la mémoire sémantique. Deux types de consolidation mnésique sont différenciés dans la littérature : d'une part la consolidation à court terme qui dure quelques secondes ou quelques minutes et qui permet le passage en mémoire à long terme, et d'autre part la consolidation à long terme qui porte sur des mois, des années ou des décennies et qui aboutit à un stockage durable des représentations mnésiques. Nous nous intéresserons essentiellement à la consolidation mnésique à long terme et au rôle du lobe temporal interne (ou LTI), qui comprend notamment l'hippocampe, dans le stockage et la récupération des souvenirs. Le premier courant théorique de la consolidation mnésique insiste sur le rôle temporellement limité du LTI dans le stockage et la récupération des événements et des informations, alors que le deuxième courant postule l'existence du rôle permanent du LTI dans la récupération des souvenirs autobiographiques. 


\subsubsection{Le modèle de Squire et Alvarez}

Squire et Alvarez (1995) ont développé l'un des premiers modèles de la consolidation à long terme pour expliquer les formes d'amnésie rétrograde avec gradient temporel observées chez des patients atteints d'une lésion du LTI. Cette structure jouerait un rôle à la fois dans l'encodage des informations et dans leur récupération pendant un certain temps. Selon cette conception, le LTI sert à indexer les multiples éléments néocorticaux qui constituent la trace mnésique de l'événement vécu. Lors des évocations successives de l'événement ou pendant le sommeil, la co-activation répétée des différents éléments néocorticaux de la trace mnésique, par l'intermédiaire du LTI, crée et renforce graduellement les interconnections néocorticales. Lorsque la consolidation est complète, les interconnections représentant l'événement sont devenues permanentes. L'évocation du souvenir s'effectue alors indépendamment du LTI. Ce mécanisme permet d'expliquer la préservation des souvenirs anciens en cas d'atteinte du LTI dans les formes d'amnésie rétrograde avec gradient temporel de Ribot (rencontrées dans la maladie d'Alzheimer débutante, voir ci-dessous), la préservation des souvenirs récents en cas d'atteinte néocorticale dans les formes avec gradient temporel inversé (observées dans la démence sémantique) et la perturbation globale des souvenirs lorsque les lésions concernent à la fois le LTI et le néocortex.

Néanmoins, ce modèle repose sur une conception de la mémoire déclarative qui ne prend pas en compte la dissociation entre les composantes épisodique et sémantique dont la pertinence a été récemment réaffirmée dans la littérature (Vargha-Khadem et al., 1997 ; Guillery et al., 2001). Ainsi, Vargha-Khadem et al. (1997) ont rapporté les cas de trois jeunes patients atteints de lésions précoces limitées à l'hippocampe. Ces patients présentaient une amnésie antérograde massive et une amnésie rétrograde autobiographique. Ils étaient incapables de rappeler le moindre événement de leur vie mais leur mémoire sémantique était bonne (connaissances sur le monde, apprentissage didactique, langage), ce qui leur avait permis de suivre un cursus scolaire pratiquement normal. Le modèle de Squire et Alvarez ne permet pas d'expliquer l'amnésie rétrograde épisodique globale observée chez les patients ayant une atteinte circonscrite au LTI, ni leur capacité à acquérir de nouvelles connaissances en mémoire sémantique malgré l'atteinte du LTI. Une approche théorique alternative a été proposée par Nadel et Moscovitch (1997) où le lobe temporal interne joue un rôle permanent dans la récupération des souvenirs autobiographiques épisodiques.

\subsubsection{Modèle du rôle permanent du LTI de Nadel et Moscovitch}

Nadel et ses collaborateurs (Nadel et Moscovitch, 1997) ont proposé un modèle alternatif de consolidation qui tient compte de la dichotomie épisodique/sémantique et de l'observation d'amnésies rétrogrades épisodiques globales chez des patients atteints d'une lésion temporale interne, ainsi que des études en neuroimagerie fonctionnelle de la mémoire autobiographique chez des sujets sains montrant l'absence d'activation différentielle de cette région cérébrale en fonction de l'intervalle de rétention (Gilboa et al., 
2004 ; Viard et al., 2007). Ces observations suggèrent, en effet, que le LTI intervient temporairement dans la récupération des informations sémantiques mais de manière permanente dans la récupération des souvenirs épisodiques. Nadel et collaborateurs proposent que le LTI et le néocortex interagissent en continu. Le stockage et la récupération en mémoire autobiographique épisodique dépendraient du LTI de façon permanente. En effet, lorsque la consolidation «standard » est complète, le LTI continue d'indexer les divers éléments de la trace mnésique dans les différentes régions cérébrales impliquées.

Dans ce contexte, nous avons élaboré une étude en IRMf dans laquelle les participants devaient rappeler des souvenirs autobiographiques en fonction de cinq périodes de vie : 3 périodes anciennes (0-17ans, 18-30 ans, 31 ans-5 dernières années) et 2 périodes récentes (5 dernières années, 12 derniers mois). Les résultats ont montré l'activation d'un réseau cérébral similaire pendant le rappel de souvenirs anciens et récents, comprenant le cortex préfrontal médian, le précunéus, le cortex cingulaire postérieur et l'hippocampe (structure faisant partie du LTI ; figure 2). Ce résultat est donc en faveur du modèle alternatif de consolidation de Nadel et Moscovitch (1997).
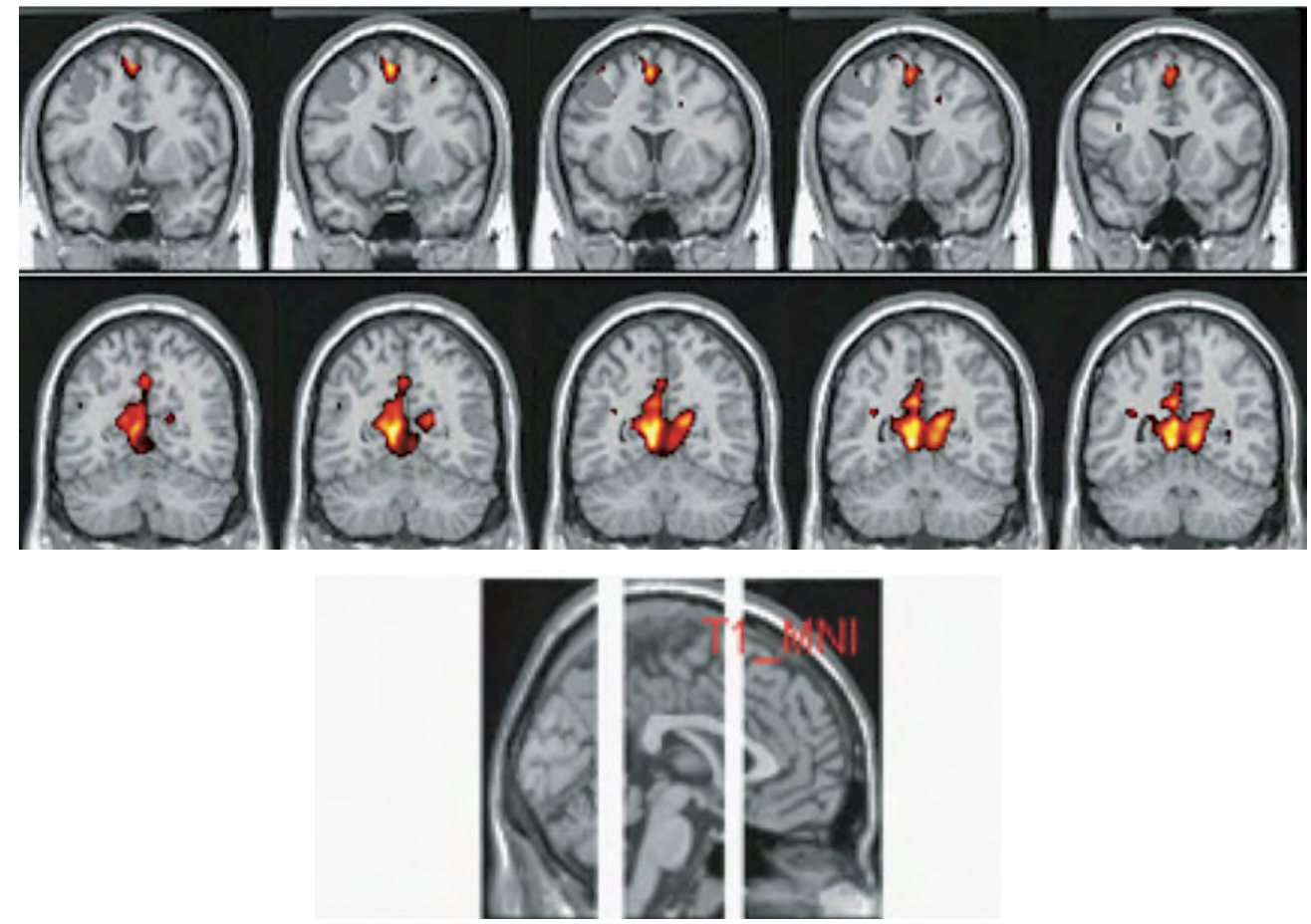

Figure 2 : Régions cérébrales activées pendant le rappel de souvenirs autobiographiques anciens et récents (cinq périodes de vie) : chez un sujet sain, le même réseau cérébral est activé quelle que soit l'ancienneté du souvenir (d'après Viard et al., 2007). 
Selon Nadel et Moscovitch (1997), le LTI intervient tout au long du stockage dans la réactivation interne de la trace mnésique de l'événement (réactualisation du souvenir) qui conduit à la création d'une trace qui sera à son tour encodée et consolidée. La répétition de ce processus produit des traces multiples liées à l'événement originel situées dans le LTI et le néocortex. Ainsi, chaque réactivation d'un souvenir crée une nouvelle trace hippocampique : les souvenirs anciens seraient donc associés par un plus grand nombre de traces mnésiques que les souvenirs récents. Gilboa et al. (2004) ont en effet montré que le rappel de souvenirs autobiographiques anciens activait l'hippocampe antérieur et postérieur, alors que le rappel de souvenirs récents activait uniquement l'hippocampe antérieur, suggérant qu'il y aurait plus de traces mnésiques lorsque les souvenirs sont anciens.

Les théories de la consolidation mettent en avant le rôle majeur du LTI et du cortex préfrontal dans le rappel autobiographique. Elles soulignent aussi l'idée qu'une atteinte quelconque du processus de récupération se traduit par une perturbation de la mémoire autobiographique.

\section{Mémoire autobiographique dans les pathologies}

\section{neurodégénératives}

\subsection{Amnésies rétrogrades et gradients temporels}

Dans la littérature, il existe trois grandes catégories d'amnésies rétrogrades: les amnésies rétrogrades présentant un gradient temporel de Ribot (préservation des souvenirs anciens au détriment des souvenirs récents), les amnésies rétrogrades avec un gradient temporel inverse (préservation des souvenirs récents au détriment des souvenirs anciens) et les amnésies rétrogrades sans gradient temporel (figure 3). Ces trois catégories d'amnésies rétrogrades sont étayées par l'étude des pathologies cérébrales localisées (LTI, lobe temporal externe....). L'étude de l'amnésie rétrograde permet d'évaluer l'influence de l'amnésie sur la dichotomie épisodique/sémantique et de mieux connaître les différents sous-systèmes mnésiques ainsi que leurs substrats neuronaux.

\subsection{Maladie d'Alzheimer}

La maladie d'Alzheimer débutante se manifeste par un déficit prépondérant de la mémoire épisodique et une atteinte du LTI. Elle se caractérise généralement par une amnésie antérograde et rétrograde. Concernant le versant antérograde, la mémoire épisodique est majoritairement perturbée alors que l'atteinte de la mémoire sémantique est variable et dépend du stade d'évolution de la maladie. L'amnésie rétrograde touche aussi bien la mémoire autobiographique que la mémoire des événements publics, quel que soit l'intervalle de rétention. 
A l'aide d'un questionnaire semi-structuré, le TEMPau, Piolino et al. (1999) ont confirmé l'atteinte de la mémoire autobiographique épisodique à un stade modéré de la démence. Le rappel total d'événements autobiographiques indique que les souvenirs anciens sont mieux préservés que les souvenirs récents, contrairement aux sujets contrôles. Sur l'ensemble des patients, $80 \%$ des souvenirs étaient génériques contre seulement $20 \%$ d'épisodiques. Ainsi, la relative préservation des souvenirs anciens chez les patients atteints de la maladie d'Alzheimer concernerait majoritairement des souvenirs sémantisés et non strictement épisodiques. Le gradient de Ribot semble refléter le gradient de sémantisation des souvenirs.

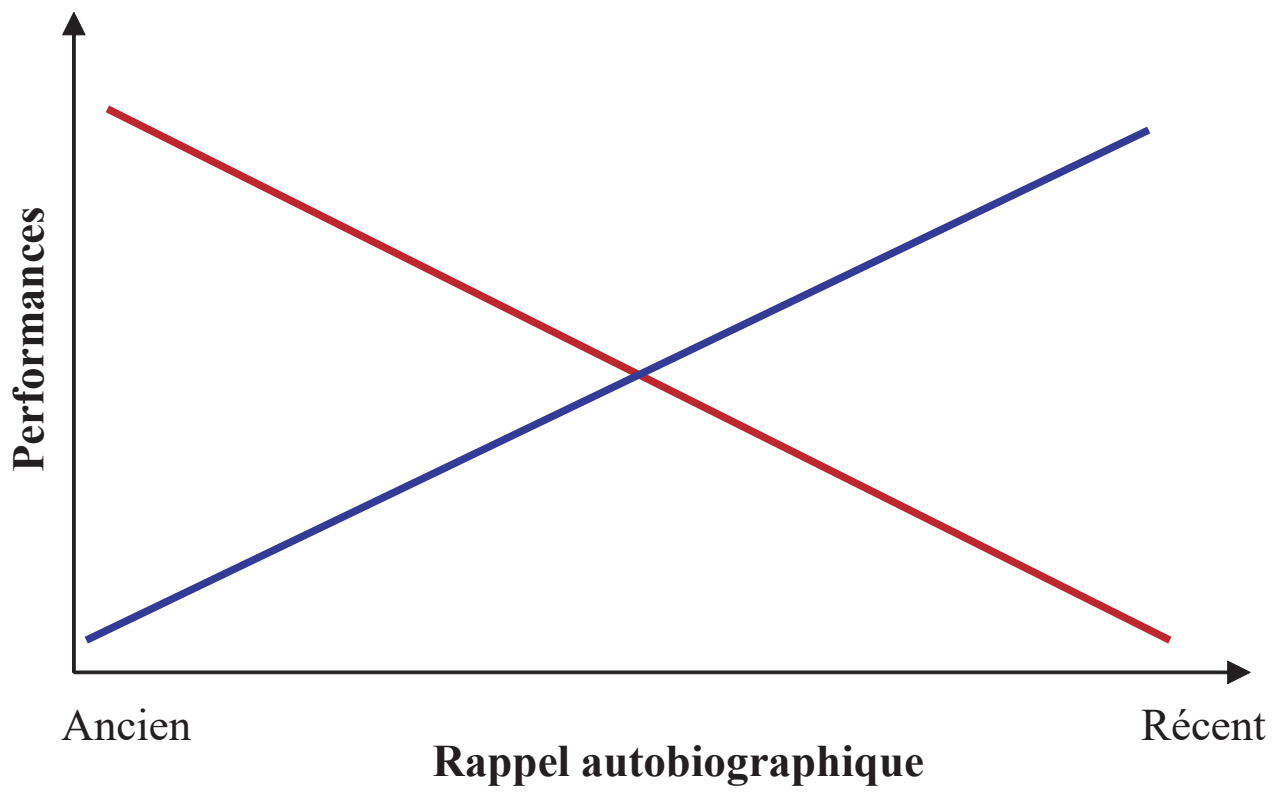

- Gradient temporel de Ribot (maladie d'Alzheimer stade léger)

- Gradient temporel inversé (démence sémantique)

Figure 3 : Rappel autobiographique suivant un gradient temporel de Ribot observé dans la maladie d'Alzheimer (rouge) et gradient temporel inversé observé dans la démence sémantique (bleu).

L'étude d'imagerie cérébrale d'Eustache et al. (2004) visait à évaluer la mémoire autobiographique de 17 patients atteints de la maladie d'Alzheimer à l'aide du questionnaire TEMPau en fonction de 3 périodes de vie (enfance/adolescence, période intermédiaire, 5 dernières années), couplée à un examen en tomographie par émissions de positions (TEP). Au niveau comportemental, les analyses ont révélé une perte de mémoire 
autobiographique suivant le gradient temporel de Ribot et une analyse qualitative a montré que les souvenirs anciens étaient plus génériques (i.e., sémantiques) que spécifiques (i.e., épisodiques). Au niveau cérébral, des analyses de corrélation ont montré le désengagement de l'hippocampe (partie du LTI) avec l'intervalle de rétention: le rappel de souvenirs récents était corrélé à l'activité de l'hippocampe, ce qui n'était pas le cas pour le rappel de souvenirs anciens. Ces analyses ont également indiqué un passage de gauche à droite de l'engagement du cortex préfrontal: le rappel de souvenirs récents était corrélé avec le cortex préfrontal droit, alors que le rappel de souvenirs anciens était corrélé avec le cortex préfrontal gauche. Les résultats de cette étude sont en accord avec les deux modèles de la consolidation évoqués précédemment : le rappel de souvenirs récents requiert le LTI, notamment l'hippocampe, mais les souvenirs anciens sémantisés ne dépendent plus que de régions néocorticales, notamment le cortex préfrontal.

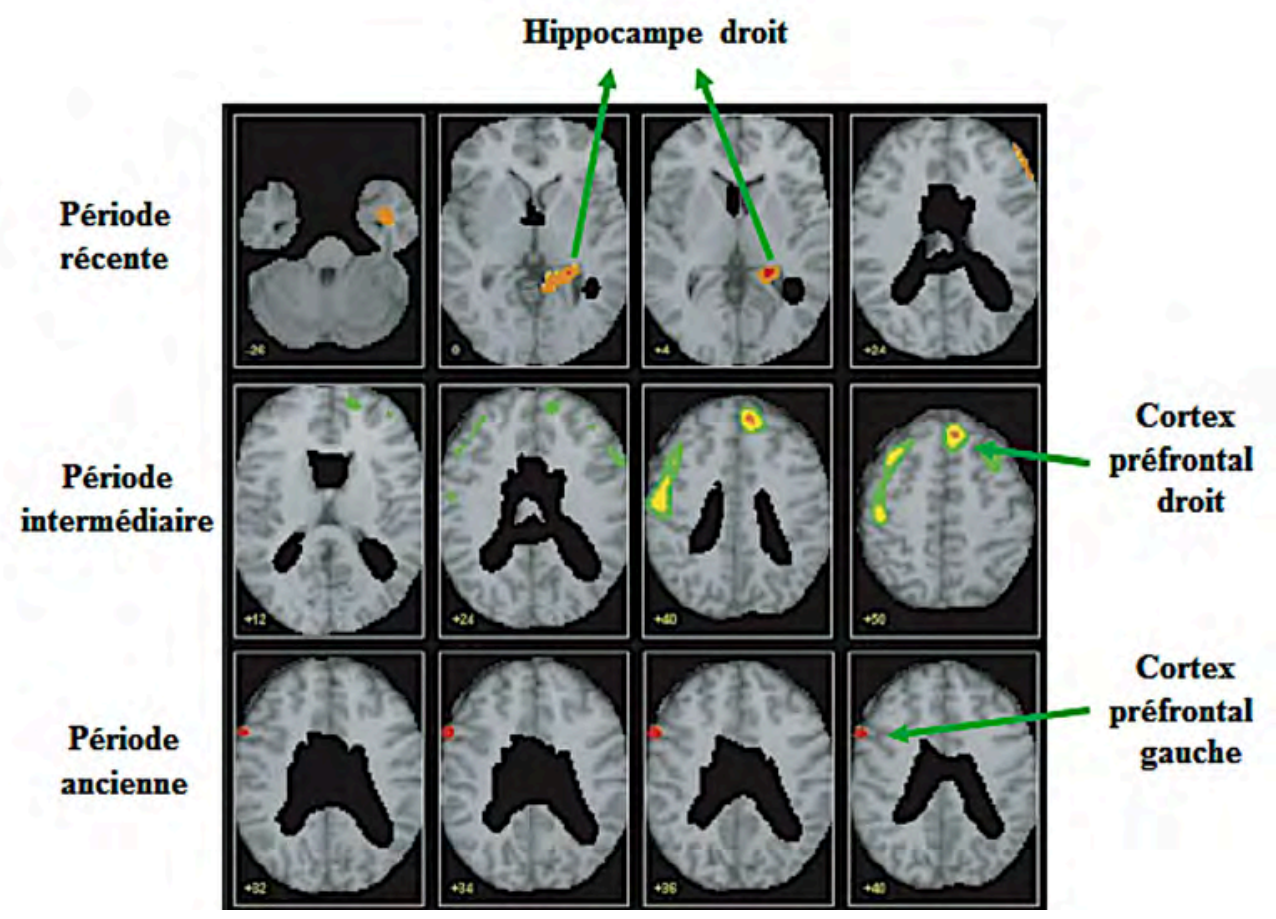

Figure 4 : Résultats d'une étude en tomographie par émissions de positions (TEP) montrant les régions cérébrales corrélées avec la performance en rappel autobiographique en fonction de 3 périodes de vie (enfance/adolescence, période intermédiaire, 5 dernières années) chez des patients atteints de la maladie d'Alzheimer (d'après Eustache et al., 2004).

\subsection{Démence sémantique}

La démence sémantique se manifeste essentiellement par un trouble de la mémoire sémantique (verbale et non verbale) alors que la mémoire épisodique, et plus généralement 
la mémoire quotidienne, sont préservées chez ces patients présentant une atrophie corticale des parties inféro-latérales du lobe temporal surtout gauche, épargnant le LTI. Cependant, de récentes études montrent que le LTI, notamment l'hippocampe, serait atrophié chez ces patients (La Joie et al., 2013 ; Viard et al., 2013). Ainsi, cette pathologie présente un profil cognitif et neuroanatomique différent de celui de la maladie d'Alzheimer débutante. Cette différence se confirme également dans la composante rétrograde puisque le profil d'amnésie autobiographique dans la démence sémantique, contrairement à la maladie d'Alzheimer débutante, ne semble pas obéir à la loi de Ribot mais présente un gradient temporel inverse où les souvenirs récents sont mieux rappelés que les souvenirs anciens.

Maguire et al. (2010) ont effectué un suivi longitudinal d'un patient atteint de démence sémantique avec un examen d'IRMf. Le patient devait ré-évoquer ses souvenirs autobiographiques dans l'IRM. Les résultats ont montré que sa mémoire autobiographique déclinait progressivement au cours des trois années consécutives qui coïncidait avec une atrophie cérébrale accrue, notamment de l'hippocampe. Dans ce même contexte, nous avons comparé le profil d'atrophie de deux patients atteints de démence sémantique au cours d'un examen d'IRMf pendant lequel les patients devaient ré-évoquer leurs souvenirs autobiographiques en fonction de cinq périodes de vie (Viard et al., 2014) : 3 périodes anciennes (0--17 ans, 18--30 ans, 31ans--5 dernières années) et 2 périodes récentes ( 5 dernières années, 12 derniers mois). Les deux patients (JPL et EP) présentaient une atrophie du cortex temporal latéral, caractéristique de cette pathologie, et le patient JPL présentait en plus une atrophie de l'hippocampe bilatéral. Au niveau comportemental, JPL avait un déficit dans le rappel de souvenirs récents et anciens, contrairement à EP qui avait des performances correctes. Au niveau des résultats d'imagerie, JPL hyperactivait certaines régions néocorticales mais ce processus de compensation restait inefficace puisqu'il n'arrivait pas à évoquer des souvenirs autobiographiques de manière épisodique. Cette étude a permis de montrer que l'intensification de l'atrophie hippocampique affecte profondément le rappel de souvenirs (récents et anciens) dans cette pathologie et que l'hyperactivation de régions néocorticales est parfois insuffisante pour compenser efficacement le déficit autobiographique chez les patients avec une atteinte hippocampique trop importante. Nous avons revu ces deux patients, ainsi que deux nouveaux patients, pour leur proposer une tâche de projection dans le futur, associée à un examen d'IRMf (voir cidessous).

\section{Projection dans le futur}

\subsection{Définition et réseau cérébral}

Selon Tulving, la mémoire épisodique nous permet de voyager mentalement dans le temps, qu'il soit passé (i.e., revivre mentalement des expériences passées) ou futur (i.e., vivre mentalement des expériences futures). Ainsi, cette mémoire épisodique nous permet de nous représenter dans le futur par projection mentale. Buckner et Carroll (2007) montrent que le réseau cérébral impliqué dans le rappel autobiographique est très similaire au réseau cérébral impliqué dans la projection vers le futur. Dans une étude en IRMf, nous 
avons demandé à des volontaires sains d'évoquer des souvenirs passés ayant eu lieu dans les 12 derniers mois et des événements futurs qu'ils avaient projeté d'effectuer dans les 12 prochains mois (Viard et al., 2011). Confortant le constat de Buckner et Carroll (2007), nos résultats d'imagerie ont montré un réseau d'activation commune entre le passé et le futur, comprenant l'hippocampe, le cortex préfrontal médian et latéral, le cortex cingulaire postérieur, le précuneus et des régions postérieures (occipitales et pariétales). Ces résultats confortent la théorie de Schacter et Addis (2007) selon laquelle la mémoire épisodique serait une base sur laquelle se construisent nos simulations futures. Ainsi, les événements passés et futurs reposent sur les mêmes informations stockées en mémoire épisodique et dépendent des même processus cognitifs (implication du soi, imagerie visuelle mentale, processus d'assemblage d'éléments disparates...).
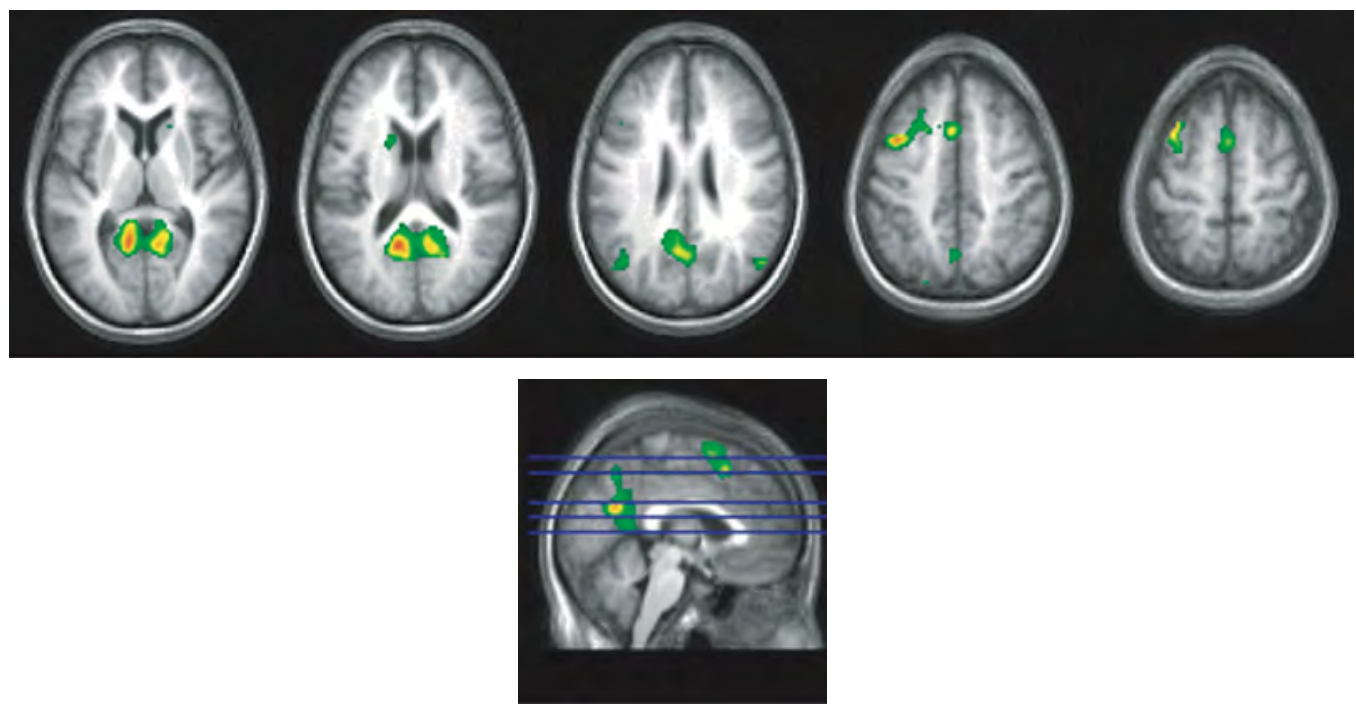

Figure 5 : Régions activées en commun lors du rappel en mémoire autobiographique et de la projection dans le futur (d'après Viard et al., 2011).

\subsection{Projection dans le futur et démence sémantique}

Peu d'études se sont intéressées à la projection dans le futur dans les pathologies neurodégénératives en imagerie cérébrale. Nous avons proposé une étude en IRMf à quatre patients atteints de démence sémantique dans laquelle ils devaient évoquer des souvenirs passés ayant eu lieu dans les 12 derniers mois et des événements futurs qu'ils avaient projeté d'effectuer dans les 12 prochains mois (Viard et al., 2014). Les résultats ont montré que tous les patients avaient une atrophie du cortex temporal latéral, caractéristique de cette pathologie. Deux patients présentaient en plus des atrophies importantes de l'hippocampe (patient JPL) et du cortex préfrontal médian (patients JPL et EP). Chez ces deux patients, l'évocation de souvenirs passés ou d'événements futurs était fortement altérée. A l'inverse, chez les patients qui ne présentaient pas d'atrophie dans ces deux régions (patient EG) ou 
une atrophie restreinte de l'hippocampe (patient LL), l'hyperactivation du tissu résiduel ou d'autres régions ayant un rôle compensatoire leur permettait de se rappeler et de se projeter efficacement dans le futur. Cette étude a permis de montrer que l'intégrité fonctionnelle de l'hippocampe et du cortex préfrontal médian était cruciale pour la projection dans le futur : l'atrophie de ces deux structures empêche cette projection alors que l'intégrité de ces deux structures, ou l'hyperactivation du tissu résiduel, permet de normaliser les capacités de projection dans le futur.

\section{Conclusion et perspectives}

Dans ce chapitre, nous avons présenté la mémoire humaine et ses troubles dans le cadre théorique actuellement le plus admis en neuropsychologie, celui des systèmes de mémoire et de leurs mécanismes de fonctionnement. Nous avons ensuite décrit le concept de mémoire autobiographique, les contributions de l'imagerie cérébrale fonctionnelle à sa compréhension, puis ses modifications dans différents cadres pathologiques. En fait, des troubles de mémoire, d'intensités diverses et avec des symptômes multiples, peuvent survenir dans des situations cliniques variées. En dehors de toute situation conduisant à une lésion cérébrale (traumatisme cranio-cérébral, accident vasculaire cérébral...), il s'agit des grandes pathologies psychiatriques comme la schizophrénie ou la dépression, mais également des pathologies non-cérébrales comme les conséquences psychosociologiques d'un cancer, le cancer du sein chez la femme ayant été le plus étudié. En fait, toute situation qui met en cause l'identité de la personne et son lien avec son environnement peut entrainer des troubles de la mémoire. Un autre exemple est le trouble de stress post-traumatique, dont les critères de diagnostic ont évolué au fil du temps et mettent aujourd'hui l'accent sur les distorsions de la mémoire, notamment des images et des pensées intrusives, qui se trouvent au cœur de la sémiologie de ce syndrome. Ainsi, au-delà des pathologies qui atteignent les mécanismes centraux de "l'instrument mémoire » et leurs substrats cérébraux, de nombreuses situations qui remettent en cause une relation harmonieuse avec soi et avec les autres sont susceptibles d'entrainer des altérations de la mémoire. Pour ces raisons, de nombreuses recherches se développent aujourd'hui aux confins des neurosciences et sciences humaines et sociales. Il en est ainsi des stéréotypes, comme ceux associés au vieillissement, qui peuvent exercer un effet significatif sur les performances de mémoire des personnes âgées, si elles sont placées dans une situation qui suggère que la tâche qu'elles réalisent est sensible aux effets de l'âge. Ces effets ne sont pas marginaux et permettent de nuancer une vision trop mécaniciste du fonctionnement de la mémoire. Un autre exemple est l'étude des interactions entre la mémoire individuelle et la mémoire collective. On connait de mieux en mieux les mécanismes qui concourent à l'élaboration de ces mémoires, mais jusqu'à présent, elles donnaient lieu à des études bien séparées, les premières relevant de la psychologie et de la biologie et les secondes de la sociologie et de l'histoire. L'approche transdisciplinaire permet de comprendre leurs interactions, mais aussi d'appréhender différemment des situations cliniques où un individu singulier éprouve des difficultés à inscrire son histoire personnelle dans l'histoire de la société dans laquelle il évolue (Legrand et al, 2015 ; Eustache et al, 2017 ; Eustache et al, 2018). La nouvelle science de la mémoire se trouve ainsi aux confins de multiples disciplines et courants de 
pensée et prend une place importante dans le monde moderne face aux nouveaux moyens de communication et aux déplacements massifs de populations.

\section{Références}

Buckner, R. L., \& Carroll, D. C. (2007). Self-projection and the brain. Trends in Cognitive Science 11:49-57.

Cabeza R, Prince SE, Daselaar SM, Greenberg DL, Budde M, Dolcos F, LaBar KS, Rubin DC (2004) Brain activity during episodic retrieval of autobiographical and laboratory events: an fMRI study using a novel photo paradigm. J Cogn Neurosci 16:1583-94.

Conway MA, Pleydell-Pearce CW (2000) The construction of autobiographical memories in the self-memory system. Psychological Review 107:261-188.

Eustache F, Amieva H, Thomas-Antérion C, Ganascia J-G, Jaffard R, Peschanski D, Stiegler B. (2017) Ma mémoire et les autres. Paris : Le Pommier.

Eustache F, Amieva H, Thomas-Antérion C, Ganascia J-G, Jaffard R, Peschanski D, Stiegler B. (2018) La mémoire au futur. Paris : Le Pommier.

Eustache F, Desgranges B. Les chemins de la mémoire. Paris: Le Pommier/Inserm; 2012.

Eustache F, Desgranges B, Lalevée C (1998) L'évaluation clinique de la mémoire. Revue neurologique 154S:18-32.

Eustache F, Faure S, Desgranges B. Manuel de Neuropsychologie. 5 ed. Paris: Dunod; 2018.

Eustache F, Guillery-Girard B. La Neuroéducation, La mémoire au cœur des apprentissages. Paris : Ed Odile Jacob, 2016.

Eustache F, Piolino P, Giffard B, Viader F, de La Sayette V, Baron JC, Desgranges B (2004) In the course of time: a PET study of the cerebral substrates of autobiographical amnesia in Alzheimer's disease. Brain 127:1549-1560.

Eustache F, Viard A, Desgranges B (2016) The MNESIS model: Memory systems and processes, identity and future thinking. Neuropsychologia 87:96-109.

Gardini S, Cornoldi C, De Beni R, Venneri A (2006) Left mediotemporal structures mediate the retrieval of episodic autobiographical mental images. NeuroImage 30:645-655.

Gilboa A, Winocur G, Grady CL, Hevenor SJ, Moscovitch M (2004) Remembering our past: functional neuroanatomy of recollection of recent and very remote personal events. Cerebral Cortex 14:1214-1225.

Greenberg DL, Rice HJ, Cooper JJ, Cabeza R, Rubin DC, Labar KS (2005) Co-activation of the amygdala, hippocampus and inferior frontal gyrus during autobiographical memory retrieval. Neuropsychologia 43:659-674.

Guillery B, Desgranges B, Katis S, de La Sayette V, Viader F, Eustache F (2001) Semantic acquisition without memories: evidence from transient global amnesia. NeuroReport 12:38653869.

La Joie R, Perrotin A, de La Sayette V, Egret S, Doeuvre L, et al. (2013) Hippocampal subfield volumetry in mild cognitive impairment, Alzheimer's disease and semantic dementia. NeuroImage Clin 3:155-162.

Legrand N, Gagnepain P, Peschanski D, Eustache F (2015) Neurosciences et mémoires collectives : les schémas entre cerveau, sociétés et cultures. Biol Aujourdhui 209:273-86 
Maguire EA, Kumaran D, Hassabis D, Kopelman MD (2010) Autobiographical memory in semantic dementia: a longitudinal fMRI study. Neuropsychologia 48:123-136.

Nadel L, Moscovitch M (1997) Memory consolidation, retrograde amnesia and the hippocampal complex. Current Opinion in Neurobiology 7:217-227.

Piolino P, Desgranges B, Giffard B, Guillery B, Benali K, Lalevée C, de La Sayette V, Eustache F (1999) La mémoire autobiographique dans le vieillissement normal et dans la maladie d'Alzheimer. Revue de Neuropsychologie 9:452-453.

Schacter DL, Addis DR (2007) The cognitive neuroscience of constructive memory: remembering the past and imagining the future. Philos Trans R Soc Lond B Biol Sci 362:773-786.

Squire LR, Alvarez P (1995) Retrograde amnesia and memory consolidation: a neurobiological perspective. Current Opinion in Neurobiology 5:169-177.

Steinvorth S, Corkin S, Halgren E. 2006. Ecphory of autobiographical memories: An fMRI study of recent and remote memory retrieval. Neuroimage 30:285-298.

Svoboda E, McKinnon MC, Levine B (2006) The functional neuroanatomy of autobiographical memory: A meta-analysis. Neuropsychologia 44:2189-2208.

Vargha-Khadem F, Gadian DG, Watkins KE, Connely A, Van Paesschen W, Mishkin M (1997) Differential effects of early hippocampal pathology on episodic and semantic memory. Science 277:376-380.

Viard A, Chételat G, Lebreton K, Desgranges B, Landeau B, et al. (2011) Mental time travel into the past and the future in healthy aged adults: an fMRI study. Brain Cogn 75:1-9.

Viard A, Desgranges B, Matuszewski V, Lebreton K, Belliard S, et al. (2013) Autobiographical memory in semantic dementia: New insights from two patients using fMRI. Neuropsychologia 51:2620-2632.

Viard A, Lebreton K, Chételat G, Desgranges B, Landeau B, Young A, De La Sayette V, Eustache F, Piolino P (2010) Patterns of hippocampal-neocortical interactions in the retrieval of episodic autobiographical memories across the entire life-span of aged adults. Hippocampus 20:153-165.

Viard A, Piolino P, Desgranges B, Chételat G, Lebreton K, et al. (2007) Hippocampal activation for autobiographical memories over the entire lifetime in healthy aged subjects: An fMRI study. Cereb Cortex 17:2453-2467.

Viard A, Piolino P, Belliard S, de La Sayette V, Desgranges B, Eustache F (2014) Episodic future thinking in semantic dementia: a cognitive and FMRI study. PLoS One 9:e111046. 
